\title{
Compatibilidade entre funções de atraso e o HCM em rodovias paulistas
}

\author{
Gustavo Riente de Andrade ${ }^{1}$, José Reynaldo A. Setti², Antônio Clóvis P. Ferraz ${ }^{3}$
}

\begin{abstract}
Resumo: Neste trabalho foram identificadas na literatura e descritas as funções de atraso mais comumente usadas nos pacotes de modelagem de demanda por transporte, a saber: (1) BPR; (2) Conical; (3) Função de Akçelik; e (4) Logística. Em seguida, foi testada a compatibilidade entre essas quatro VDFs e os modelos atuais do HCM, calibrados para o ambiente rodoviário brasileiro. Como principais conclusões, tem-se que os melhores ajustes foram obtidos com o uso da função do BPR e logística. Por outro lado, as funções Conical e de Akçelik devem ser empregadas com cautela, pois tendem a subestimar o atraso em condições de fluxos de tráfego baixos a médios, comuns em rodovias rurais. Especificamente com relação às rodovias de pista simples, a função logística se mostrou interessante, devido a capacidade de refletir atrasos mais acentuados para baixos fluxos de tráfego, e produzir maiores atrasos em condições congestionadas, característica desejável em modelagem de demanda.
\end{abstract}

Palavras-chave: Rodovias, HCM, Funções de Atraso.

\begin{abstract}
This paper identified in the literature and described the most commonly used delay functions in transportation demand modeling packages: (1) BPR; (2) Conical; (3) Akçelik function; and (4) Logit-Based. Then it was tested the compatibility between these four VDFs and the current HCM models, calibrated to Brazilian highways. As the main conclusions, it was observed that the best fits were obtained using the BPR and the Logit-Based Functions. On the other hand, the Conical and Akçelik functions should be used with caution, since they tend to underestimate the delay for low to moderate traffic flows, common in rural highways. Specifically with respect to two-lane highways, the Logit-Based function has proved to be of interest, because of its capability to reflect more severe delays for low traffic flows, while producing greater delays for congested conditions, which is a desirable characteristic in demand modeling.
\end{abstract}

Keywords: Highways, HCM, Volume-Delay Functions

\section{INTRODUÇÃO}

Funções de atraso, também conhecidas como VDFs (Volume-Delay Functions, do inglês), são relações matemáticas usadas pela etapa de alocação de tráfego dos modelos de previsão de demanda, para levar em conta o efeito do aumento do fluxo de tráfego no tempo gasto para percorrer cada rota possível entre as diversas origens e destinos de viagens. De forma geral, as funções incluídas nos pacotes de modelagem de demanda disponíveis no mercado têm parâmetros que podem ser ajustados pelo usuário, de forma a se obter resultados mais condizentes com a realidade em estudo.

Essa possibilidade, embora essencial para o correto desenvolvimento de um estudo, deve ser usada com cuidado, devido ao risco de se adotar parâmetros de calibração que, embora aceitos pelos modelos, reproduzam tempos de viagem inconsistentes com o que seria realmente observado.

Com efeito, uma VDF reflete a relação entre fluxo de tráfego e tempo de viagem. Sendo o tempo inversamente proporcional à velocidade média da corrente de tráfego, tem-se que as funções de atraso são análogas às relações

\footnotetext{
${ }^{1}$ Gustavo Riente de Andrade, Departamento de Transportes, Escola de Engenharia de São Carlos, Universidade de São Paulo. (guriente@hotmail.com)

2 José Reynaldo A. Setti, Departamento de Transportes, Escola de Engenharia de São Carlos, Universidade de São Paulo. (jrasetti@usp.br)

${ }^{3}$ Antônio Clóvis P. Ferraz, Departamento de Transportes, Escola de Engenharia de São Carlos, Universidade de São Paulo. (coca@sc.usp.br)
}

Manuscrito recebido em 05/06/2015 e aprovado para publicação em 29/08/2015

Este artigo é parte de TRANSPORTES v. 23, n. 3, 2015. ISSN: 2237-1346 (online). DOI: 10.14295/transportes.v23i3.936 fluxo-velocidade, descritas pela literatura de engenharia de tráfego desde o modelo pioneiro de Greenshields.

Atualmente, as relações fluxo-velocidade rodoviárias mais utilizadas no Brasil são as apresentadas pelo Highway Capacity Manual - HCM, que é usado pelos órgãos e profissionais no Brasil como referência para estudos de capacidade e avaliação da qualidade do serviço em rodovias (Setti, 2009; Andrade et al., 2011).

\subsection{Meta e objetivos específicos}

Este trabalho se insere em um projeto de pesquisa mais amplo, que busca criar compatibilidade de modelos de engenharia de transportes usados para o planejamento e operação de rodovias brasileiras. Nesse contexto, a meta deste estudo é investigar a interface entre as funções de atraso usadas por modelos de previsão de demanda e as relações fluxo-velocidade do HCM. Para isso, são estabelecidos os seguintes objetivos específicos:

- Investigar as várias funções de atraso disponíveis na literatura, para o caso de trechos homogêneos rodoviários;

- Ajustar as relações disponíveis aos modelos do HCM calibrados para rodovias paulistas, produzindo valores default para os parâmetros de calibração de dada VDF; e

- Discutir a adequabilidade dos modelos ajustados em cada caso.

\section{FUNÇÕES DE ATRASO PARA TRECHOS HOMOGÊNEOS RODOVIÁRIOS}

As funções de atraso (VDFs) são usadas para se calcular um fator de atraso em relação à velocidade de fluxo 
livre. Trata-se de um fator multiplicativo $f(x)$, que é aplicado ao tempo de viagem em condições de fluxo livre, sendo $f(x)=f(V / C)$. Nessas equações, $V$ representa a demanda em termos do fluxo de tráfego, normalmente dada em veículos equivalentes por hora por faixa, enquanto que $C$ é a capacidade da seção, na mesma unidade usada para descrever $V$.

Os pacotes de modelagem de demanda disponíveis no mercado incorporam uma série de funções de atraso préprogramadas, que contam com parâmetros de calibração ajustáveis pelo usuário, embora valores default sejam fornecidos. Além disso, várias dessas ferramentas também permitem que o usuário programe e use sua própria VDF, o que pode indicar que a indústria de software de modelagem reconhece a necessidade de ajustes locais e melhorias aos modelos existentes (Huntsinger e Rouphail, 2011).

Em qualquer caso, qualquer VDF deve obedecer uma série de requisitos de modo a serem consideradas adequadas ao uso em modelos de demanda (Spiess, 1990), em especial:

1. $f(V / C)$ deve ser monotônica e crescente. Essa condição é necessária para a convergência dos métodos de alocação de tráfego;

2. $f(0)=1$;

3. $f^{\prime}(V / C)$ deve existir e ser crescente. Essa condição garante que a curva de atraso seja convexa, o que é uma propriedade desejável para VDFs (Huntsinger e Rouphail, 2011).

A seguir, serão descritas as principais funções de atraso encontradas na literatura, que já foram incorporadas à maioria dos modelos de demanda existentes no mercado.

\subsection{Função de atraso do Bureau of Public Roads - BPR}

A função de atraso do Bureau of Public Roads - BPR é provavelmente a mais antiga das VDFs disponíveis para o uso e, ainda assim, a mais usada. A função BPR (BPR, 1964) se baseia na segunda edição do Highway Capacity Manual - HCM (HRB, 1965) e possui formato parabólico, sendo sua formulação:

$$
f\left(\frac{V}{C}\right)=\left[1+\alpha\left(\frac{V}{C}\right)^{\beta}\right]
$$

em que $\alpha$ e $\beta$ são parâmetros de calibração, sendo $\beta>$ 1 , para garantir a forma convexa da curva, permitindo valores crescentes de atraso em condições de demanda próxima à capacidade.

Historicamente, foram estabelecidos valores default de 0,15 para $\alpha$ e 4 para o $\beta$ (Caliper, 2013), embora valores de 2 a 12 para $\beta$ sejam usados na prática (Spiess, 1990). Quanto maior for $\beta$, menor o atraso para fluxos de tráfego baixos e o efeito do congestionamento se torna mais tardio e súbito. A função BPR tende a subestimar o atraso para o caso de fluxo congestionado, quando $V / C>1$ (Singh e Dowling, 2002).

Um estudo recente (Machado e Ribeiro, 2003) calibrou a função do BPR para uma via no Brasil a partir de dados empíricos. No caso, estudou-se um trecho da ponte Rio-Niterói, obtendo-se $\alpha=0,21$ e $\beta=3,82$, considerados pelos autores como próximos aos originais.

\subsection{Função de atraso Conical}

Com o objetivo de superar uma eventual distorção da função do BPR quanto à superestimação da velocidade em condições de congestionamento, Spiess (1990) desenvolveu uma nova VDF, nomeada a partir de então de função Conical, que possui a seguinte formulação:

$$
f\left(\frac{V}{C}\right)=2+\sqrt{\alpha^{2}\left(1-\frac{V}{C}\right)^{2}+\beta^{2}}-\alpha\left(1-\frac{V}{C}\right)-\beta
$$

em que:

$$
\beta=\frac{2 \alpha-1}{2 \alpha-2}
$$

sendo $\alpha$ um parâmetro de calibração tal que $\alpha>1$.

O desenvolvimento da função Conical original não usou dados empíricos para a calibração. O objetivo foi buscar uma relação matemática baseada em geometria básica e álgebra, que tivesse um formato similar à do BPR, mas produzisse resultados capazes de levar à convergência mais rápida e melhor ajustada dos modelos de alocação de tráfego, em especial em redes nas quais o efeito do congestionamento tem um papel central. Por fim, o próprio autor recomenda que seja realizada pesquisa adicional e calibração a fluxos e velocidades observados para adequar o uso desta função às condições locais.

\subsection{Função de atraso de $A k c ̧ e l i k$}

Também desenvolvida para a modelagem do tráfego em condição de fluxo congestionado e não congestionado, a VDF conhecida como função de Akcelik (Akçelik, 2003) usa um modelo baseado em teoria das filas para o cálculo do atraso em condições de fluxo não-interrompido, similar ao do HCM 2000 (TRB, 2000), e uma relação determinística para condições de saturação (Singh e Dowling, 2002).

Com isso, para obter-se o fator de atraso por km, e partindo-se de condições sem filas em trechos de fluxo não interrompido, é usada a seguinte equação:

$$
f\left(\frac{V}{C}\right)=\left\{\begin{array}{l}
1, \text { se } \frac{V}{C} \leq \frac{B P}{C} ; \\
\left(3600 \frac{1}{F F S}\right)+900 \cdot T_{f}\left[\left(\frac{V}{C}-1\right)+\sqrt{\left.\left(\frac{V}{C}-1\right)^{2}+8 J \cdot \frac{\left(\frac{V}{C}-\frac{B P}{C}\right)}{T_{f} \cdot C}\right]}\right.
\end{array}\right.
$$

em queFFS: velocidade de fluxo livre $[\mathrm{km} / \mathrm{h}]$;

$T_{f}$ :duração do período de análise, dentro da hora de projeto [0,25 h, no HCM];

$B P$ :ponto de transição preconizado pelo HCM (TRB, 2000; TRB, 2010) para autoestradas e rodovias de pista dupla, a partir do qual a velocidade passa a decrescer em função do aumento no fluxo de tráfego, [veíc/(h.faixa)]

J:parâmetro de calibração do modelo, função de outras variáveis da relação fluxo-velocidade, como se segue:

$$
J=2 C\left(\frac{\left(\frac{F F S}{C S}-1\right)^{2}}{\left(F F S^{2} T_{f}\left(1-\frac{B P}{C}\right)\right)}\right)
$$

sendo $C S$ a velocidade na capacidade. Como uma das vantagens da função de Akçelik quanto aos objetivos deste 
trabalho, tem-se que é possível sua calibração direta a partir da relação com os parâmetros da relação fluxo-velocidade do HCM, como mostrado nas Equações 4e 5.

\subsection{Função de atraso logística}

Além das VDFs descritas anteriormente, alguns pacotes de modelagem de demanda (Caliper, 2013) incorporaram uma nova função, baseada em um modelo Logit. O componente da equação usado para se calcular o atraso em tramos de uma rede tem a seguinte formulação:

$$
f\left(\frac{V}{C}\right)=c_{1}\left(1-\frac{c_{2}}{1+\exp \left(c_{3}-c_{4} V / C\right)}\right)^{-1}
$$

em que $C_{1}, C_{2}, C_{3}$ e $C_{4}$ são parâmetros de calibração.

Como resultado, a curva conhecida como logística tem dois pontos de inflexão, que lhe conferem o formato típico em “S”. Isso permite a variação do aumento marginal do atraso a medida em que o fluxo de tráfego aumenta, o que pode ser potencialmente útil para a diferenciação da velocidade entre regimes de tráfego ou mesmo entre diferentes porções da curva fluxo-velocidade.

\subsection{Considerações finais}

Neste capítulo, foram descritas as principais funções de atraso (VDFs) que são mais comumente referenciadas na literatura e incorporadas nos pacotes de modelagem de demanda disponíveis no mercado, bem como sua relação com o modelo fluxo-velocidade do HCM.

Verificou-se que a função do BPR (BPR, 1964), a mais antiga VDF, ainda é reconhecidamente a mais usada, mesmo que análoga ao modelo do HCM 1965 (HRB, 1965) para autoestradas. Ao longo das décadas, novos modelos foram sendo desenvolvidos, em grande parte para atender à necessidade de se estimar atrasos para condições de demanda acima da capacidade, em especial em redes urbanas.

Nesse esforço, incluiu-se a função Conical (Spiess, 1990). Posteriormente, um estudo conduzido por Kurth et al. (1996) com base no HCM de 1994 (TRB, 1994) mostrou que, com a caracterização adequada dos tramos da rede de simulação e ajuste adequado das VDFs usadas aos modelos do HCM, é possível obter um bom ajuste em termos de velocidades e fluxos de tráfego mesmo em condições de congestionamento (Huntsinger e Rouphail, 2011).

Após a publicação da quarta edição do HCM (TRB, 2000), foi desenvolvida a função de Akçelik, que busca manter um desejável grau de compatibilidade com os parâmetros usados pelo manual de capacidade. Foi incorporado inclusive o conceito de $B P$, o ponto de transição a partir do qual a velocidade passa a decrescer em função do aumento no fluxo de tráfego, em autoestradas e rodovias de pista dupla. Por fim, tem-se na função Logística uma alternativa a qual, embora pouco explorada na literatura consultada, se encontra implementada em pacotes disponíveis no mercado.

No próximo capítulo, será analisada e discutida a compatibilidade entre as várias funções de atraso descritas neste item e os modelos para trechos de fluxo não interrompidos do HCM, calibrados para rodovias do estado de São Paulo para autoestradas e rodovias de pista dupla (Andrade e Setti, 2014) e rodovias de pista simples (Bessa Jr. e Setti, 2011).

\subsection{Funções de Atraso em Rodovias}

Neste item, as várias funções de atraso descritas anteriormente foram comparadas aos modelos do HCM adaptado às condições das rodovias paulistas. Foram consideradas nessa análise:

- Autoestradas e rodovias de pista dupla rurais e urbanas, conforme recomendado por Andrade e Setti (2014); e

- Rodovias de pista simples, usando-se o modelo calibrado por Bessa Jr. e Setti (2011).

\subsection{Relações fluxo-velocidade usadas}

Os métodos de alocação de tráfego usados pelos modelos de previsão de demanda, de forma geral, consideram volumes de tráfego conforme informados pelo usuário. Quando métodos de alocação multiclasse são empregados, a aplicação de fatores de equivalência para os veículos pesados é feita normalmente sobre os dados da demanda, de maneira direta (Caliper, 2013). No entanto, outros fatores de correção com relação às características da via devem ser estabelecidos tramo a tramo da rede. A seguir, será mostrada a relação entre a razão $V / C$ e o atraso para os tipos de rodovia estudados, sendo aplicados os fatores de ajuste conforme o caso.

\subsubsection{Autoestradas e rodovias de pista dupla}

De acordo com o método do HCM (TRB, 2010), o fluxo equivalente tráfego $v$, em cpe/(h.faixa), em autoestradas e rodovias de pista dupla, é calculado como:

$$
v=\frac{V}{P H F \cdot N \cdot f_{H V} \cdot f_{p}}
$$

em que

$V$ : volume de tráfego total [veíc/h];

PHF: fator de pico horário;

$N$ : número de faixas;

$f_{H V}$ : fator de ajuste quanto à equivalência dos veículos pesados [cpe/(h.faixa)]; e

$f_{p}$ : fator quanto ao efeito da familiaridade da população de motoristas com a via.

Para o caso estudado, é analisado o efeito do fluxo sobre o atraso por faixa, em condições padrão. Assim, $N$, $P H F$ e $f_{p}$ são definidos como 1. Além disso, como observado anteriormente, a conversão dos veículos pesados em veículos equivalentes em métodos de alocação de tráfego é normalmente feita sobre a demanda, de forma que para a construção da VDF para condições padrão, $f_{H V}$ é definido como 1 e, consequentemente, $v=V$.

O tempo de viagem, por sua vez, é calculado como inverso da velocidade, segundo os modelos propostos por Andrade e Setti (2014) para rodovias de pista dupla rurais (Equação 8) e urbanas (Equação 9): 


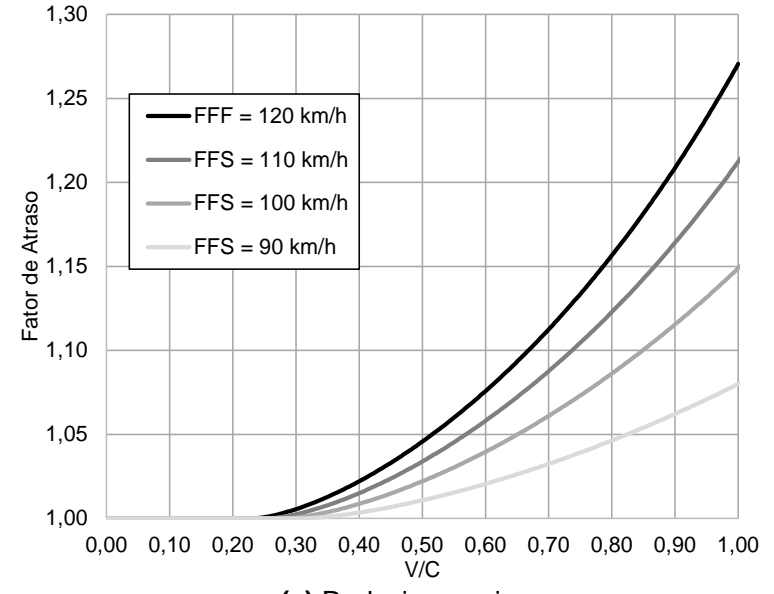

(a) Rodovias rurais

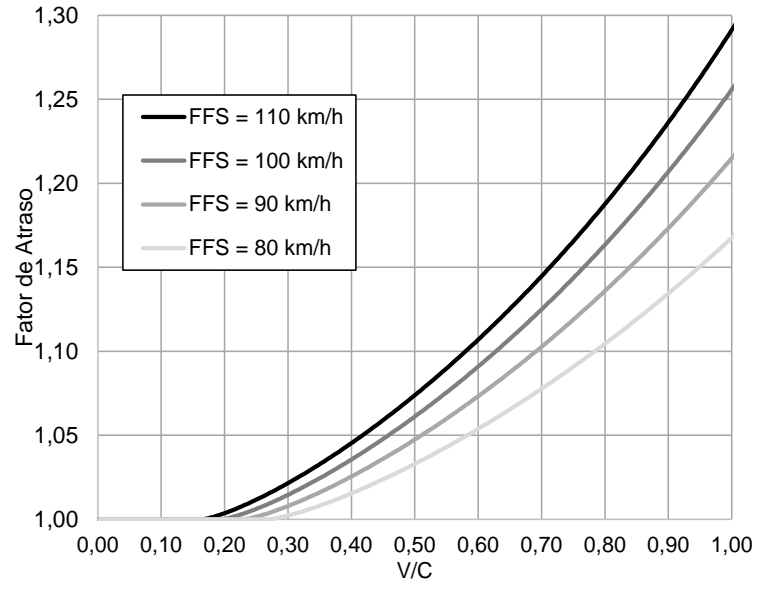

(b) Rodovias urbanas

Figura 1. Modelos de atraso propostos para rodovias de pista dupla

$S=F F S$, se $\quad v \leq-7,5 F F S+1400$

$$
\begin{aligned}
& S=F F S-\left(F F S-\frac{C}{26}\right) \times\left[\frac{v-(-7,5 F F S+1400)}{C-(-7,5 F F S+1400)}\right]^{1,5} \\
& C=12,5 F F S+1000 .
\end{aligned}
$$

$$
\begin{aligned}
& S=F F S \text {, se } \quad v \leq-3,75 F F S+835 \\
& F F S-\left(F F S-\frac{C}{25}\right) \times\left[\frac{v-(-3,75 F F S+835)}{C-(-3,75 F F S+835)}\right]^{1,3} \\
& C=17 F F S+380
\end{aligned}
$$

A partir da análise das Equações 7 a 9, é possível verificar que o atraso varia conforme a velocidade de fluxo livre FFS. Assim, a Figura 1 apresenta curvas de atraso para rodovias de pista dupla paulistas rurais e urbanas, para diferentes valores de FFS. Nota-se que os atrasos são sistematicamente maiores nas rodovias urbanas, para valores iguais de $V / C$. Além disso, em ambos os casos, quanto menor a velocidade de fluxo livre, menor o atraso. Isso ocorre porque a diferença entre FFS e a velocidade na capacidade é tanto menor quanto menor for FFS, de forma que o atraso relativo cresce com o aumento da velocidade inicial FFS.

\subsubsection{Rodovias de pista simples}

O cálculo do volume equivalente em rodovias de pista simples segue uma formulação similar à Equação 7.

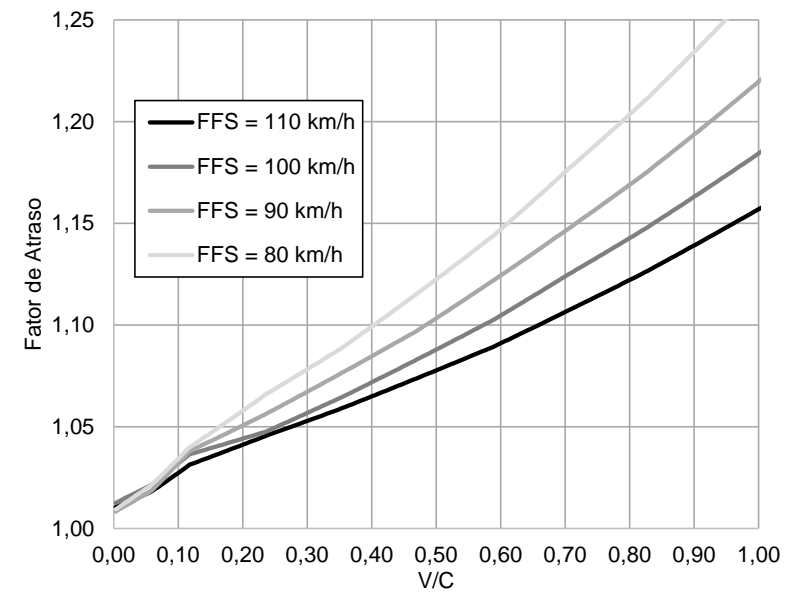

(a) Terreno plano
No entanto, é aplicado um fator relacionado à geometria da via $f_{g}$ :

$$
v=\frac{V}{P H F \cdot f_{g} \cdot f_{H V}}
$$

sendo as demais variáveis conforme definidas anteriormente. Para trechos homogêneos genéricos, o HCM preconiza valores de $f_{g}$ para terreno plano e ondulado, em função de vários intervalos de fluxo de tráfego equivalente (TRB, 2010). Assim, adotando-se PHF e $f_{H V}$ igual a 1 (condições base), tem-se que o volume de tráfego a ser alocado é calculado como $V=v \cdot f_{g}$, sendo necessária a aplicação desse fator às curvas de atraso usadas para balizar as VDFs a serem ajustadas. Neste trabalho, foram adotados os valores de $f_{g}$, ATS obtidos por Setti et al. (2011).

Para o cálculo da velocidade média de viagem ATS, tomou-se como base o modelo linear unidirecional do HCM (TRB, 2010), adaptado por Bessa Jr. e Setti (2011) para o formato:

$$
\text { ATS }=F F S+a_{1} \cdot v_{d}+a_{2} \cdot v_{o}-f_{n p, A T S}
$$

em que:

$a_{1}$ e $a_{2}$ :parâmetros de calibração;

$v_{d}$ :tráfego no sentido analisado [cpe/h];

$v_{o}$ :tráfego no sentido oposto [cpe/h]; e

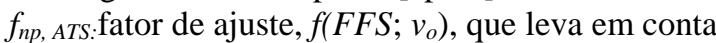
o efeito das zonas de proibição de ultrapassagem (NPZ) na

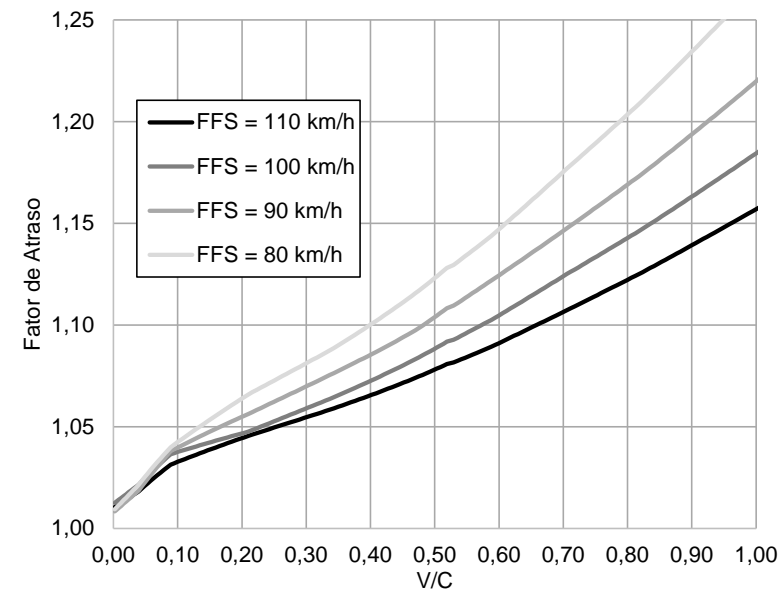

(b) Terreno ondulado

Figura 2. Funções de atraso propostas para rodovias de pista simples 


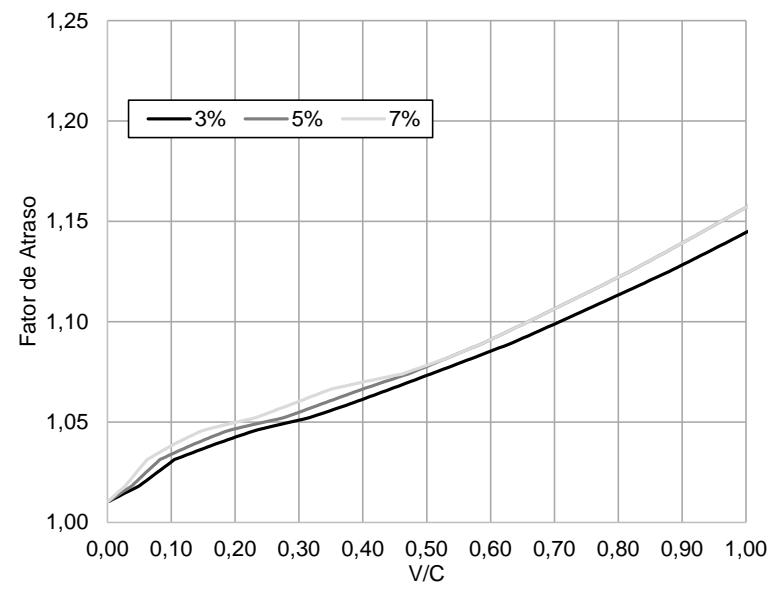

(a) FFS $=110 \mathrm{~km} / \mathrm{h}$

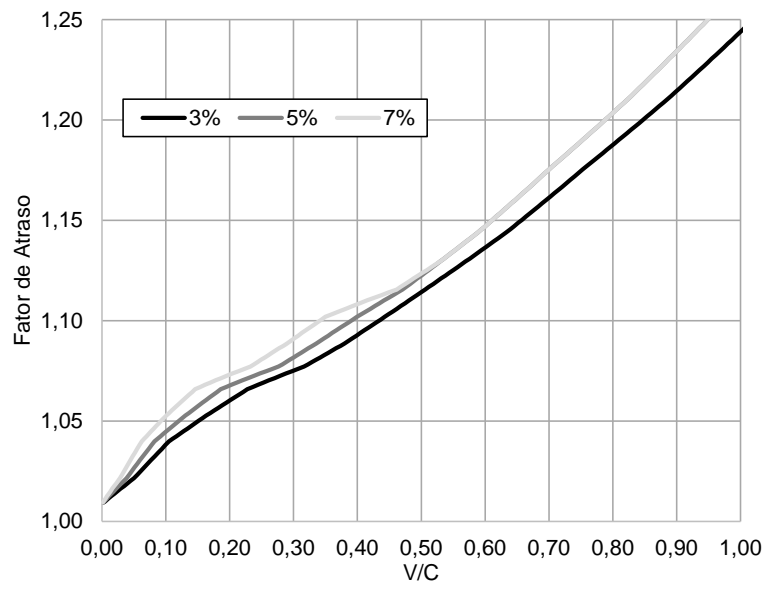

(b) $\mathrm{FFS}=80 \mathrm{~km} / \mathrm{h}$

Figura 3. Funções de atraso propostas para rodovias de pista simples

velocidade média [km/h], estabelecido neste estudo conforme proposto por Setti et al. (2011).O NPZ por sua vez foi definido como $20 \%$ para terreno plano e $50 \%$ para terreno ondulado, conforme recomendação do HCM (TRB, 2010).

Para trechos rodovias em terreno montanhoso, o HCM não fornece valores de $f_{g}$. Nesse caso, o manual indica o uso dos fatores estabelecidos para rampas específicas, e um percentual de zonas de proibição de ultrapassagem (NPZ) default de $80 \%$. A Figura 3

Neste trabalho, foi considerada uma divisão padrão entre os sentidos de 50/50, tendo-se $v_{d}=v_{o}$. Com base nas condições impostas pelas Equações 10 e 11, foram estabelecidas curvas de atraso para diferentes velocidades de fluxo livre e para terreno plano e ondulado, obtendo-se os resultados mostrados nas Figuras 2 e 3. As relações obtidas são próximas entre os tipos de geometria, variando ligeiramente até $V / C$ em torno de 0,15 , em função da aplicação de diferentes valores de $f_{g}$. Ao contrário do que ocorre para as rodovias de pista dupla, menores valores de FFS estão ligados a atrasos relativos crescentes. Além disso, embora os fatores de atraso em condições próximas à capacidade $(V / C=$ 1) sejam similares aos das rodovias duplicadas, destaca-se que há aumentos nos tempos de viagem mesmo para valores baixos de tráfego, característica intrínseca de rodovias de pista simples.

\section{COMPATIBILIDADE DAS VDFS EXISTENTES E O HCM}

Nesta etapa do trabalho, as funções de atraso apresentadas no capítulo 2 foram ajustadas às curvas de atraso do HCM. Para tanto, foi estimado o fator de atraso médio para intervalos de 10 cpe/(h.faixa), usando-se cada modelo a ser comparado.

Em seguida, foi empregado um algoritmo de otimização não linear (GRG - Generalized Reduced Gradient), cujo objetivo é a minimização do quadrado dos erros entre os fatores de atraso previstos por cada um dos modelos testados e os valores obtidos com o uso dos modelos do HCM calibrados para rodovias paulistas, obedecendo-se às condições:

- Para a função BPR, $\beta$ deve ser maior do que 1 , como citado anteriormente;

- Para a função Conical, $\alpha$ deve ser maior do que 1; e

Tabela 1. Parâmetros de calibração e erro quadrático por VDF e FFS - rodovias de pista dupla

\begin{tabular}{|c|c|c|c|c|c|c|c|c|}
\hline \multirow{2}{*}{$\begin{array}{l}\text { Parâmetro } \\
F F S\end{array}$} & \multicolumn{3}{|c|}{ Rodovias de pista dupla rurais } & \multicolumn{5}{|c|}{ Rodovias de pista dupla urbanas } \\
\hline & 120 & 110 & 100 & 90 & 110 & 100 & 90 & 80 \\
\hline \multicolumn{9}{|c|}{ Função do BPR } \\
\hline$\alpha$ & 0,27 & 0,22 & 0,15 & 0,08 & 0,29 & 0,26 & 0,22 & 0,17 \\
\hline$\beta$ & 2,57 & 2,64 & 2,71 & 2,79 & 2,02 & 2,12 & 2,23 & 2,38 \\
\hline Erro & 0,003 & 0,002 & 0,002 & 0,001 & 0,000 & 0,002 & 0,002 & 0,003 \\
\hline \multicolumn{9}{|c|}{ Função Conical } \\
\hline$\alpha$ & 46 & 96 & 220 & 513 & 31 & 47 & 80 & 147 \\
\hline Erro & 2,191 & 1,768 & 1,313 & 1,013 & 2,501 & 2,125 & 1,718 & 1,325 \\
\hline \multicolumn{9}{|c|}{ Função de Akçelik } \\
\hline$J$ & 0,135 & 0,098 & 0,058 & 0,021 & 0,155 & 0,140 & 0,119 & 0,089 \\
\hline Erro & 1,80 & 1,12 & 0,58 & 0,18 & 2,30 & 1,64 & 1,07 & 0,60 \\
\hline \multicolumn{9}{|c|}{ Função Logística } \\
\hline$C_{1}$ & 0,96 & 0,97 & 0,98 & 0,99 & 0,97 & 0,98 & 0,99 & 0,99 \\
\hline$C_{2}$ & 1,33 & 1,29 & 1,25 & 1,08 & 0,35 & 0,3 & 0,25 & 0,19 \\
\hline$C_{3}$ & 3,92 & 4,22 & 4,66 & 5,27 & 2,91 & 3,19 & 3,55 & 3,99 \\
\hline$C_{4}$ & 2,45 & 2,56 & 2,69 & 2,84 & 3,75 & 4,16 & 4,63 & 5,21 \\
\hline Erro & 0,010 & 0,007 & 0,004 & 0,001 & 0,002 & 0,002 & 0,002 & 0,001 \\
\hline
\end{tabular}




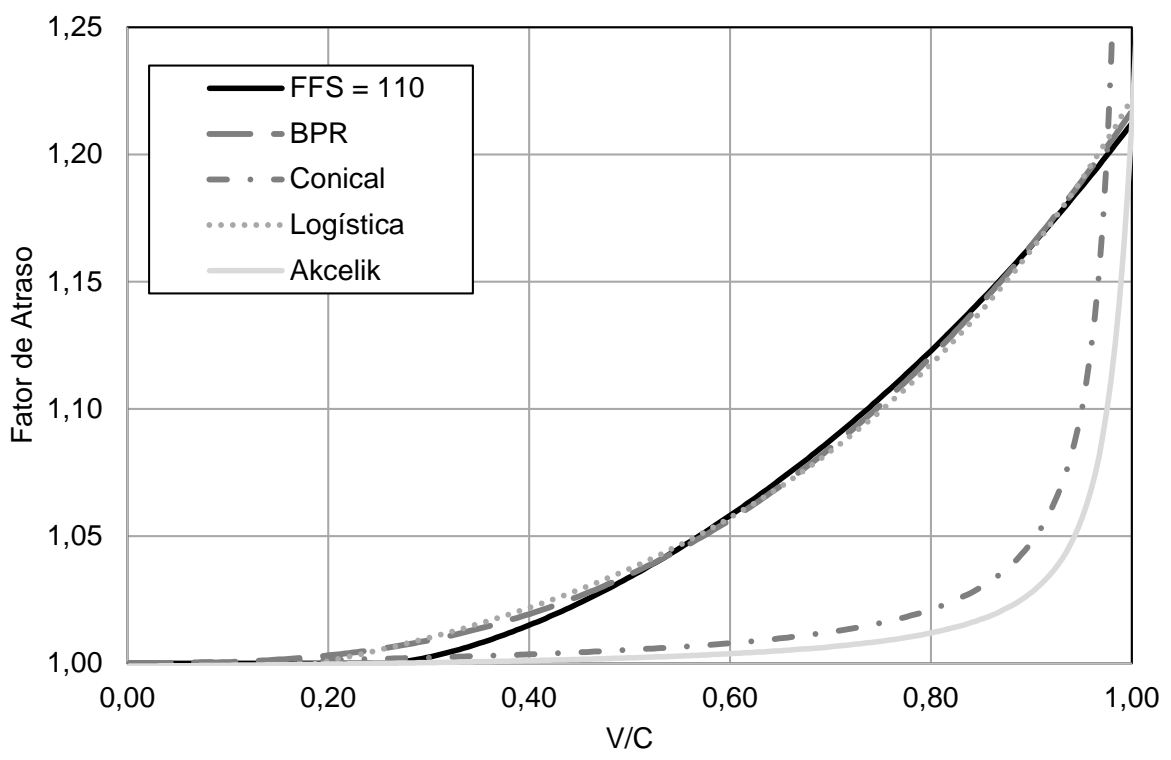

Figura 4. Comparação das funções de atraso para uma rodovia de pista dupla rural de FFS $=110 \mathrm{~km} / \mathrm{h}$

- Para a função de Akçelik, não é usado o método de otimização, sendo a função ajustada por meio do parâmetro $J$ que, por relaciona-se automaticamente às variáveis do modelo do HCM.

A seguir, são apresentados os resultados produzidos em cada caso, em termos dos parâmetros de calibração obtidos, bem como a qualidade do ajuste, medida por meio da soma do erro quadrático de cada caso.

\subsection{Ajuste para rodovias de pista dupla}

A Tabela 1 apresenta os parâmetros dos modelos de VDF descritos no Capítulo 2, obtidos para as rodovias de pista dupla rurais e urbanas através do método descrito. Tais parâmetros podem ser usados em estudos de modelagem de demanda por transporte na falta de uma calibração local da função de atraso escolhida.

Comparando-se os erros ligados a cada modelo, notase que, apesar de ser um modelo antigo, a função do BPR, juntamente com a logística, foi aquela que produziu os melhores ajustes ao modelo do HCM calibrado às rodovias paulistas.
A Figura 4 mostra essa comparação na forma de um gráfico para o caso de uma típica rodovia de pista dupla rural de velocidade de fluxo livre de $110 \mathrm{~km} / \mathrm{h}$. Destaca-se o bom ajuste das funções do BPR e logística. As funções Conical e de Akçelik, por outro lado, embora sejam baseadas originalmente na estrutura parabólica do próprio HCM, deram especial enfoque à produção de valores mais altos de atraso em condições próximas à capacidade e congestionadas. Em vista dessa característica, a solução de menor erro quadrático subestima o atraso para fluxos de tráfego médios ou baixos.

Dessa forma, é recomendado que as funções Conical e de Akçelik sejam usadas com cautela para a modelagem da demanda em rodovias rurais, para as quais a relação entre o fluxo de tráfego e o atraso em condição não saturadas é especialmente relevante e os congestionamentos, menos frequentes do que em vias urbanas arteriais ou coletoras.

\subsection{Ajuste para rodovias de pista simples}

Como para o caso das rodovias de pista dupla, a Tabela 2 apresenta os parâmetros de calibração obtidos para as rodovias de pista simples em terreno plano ou ondulado,

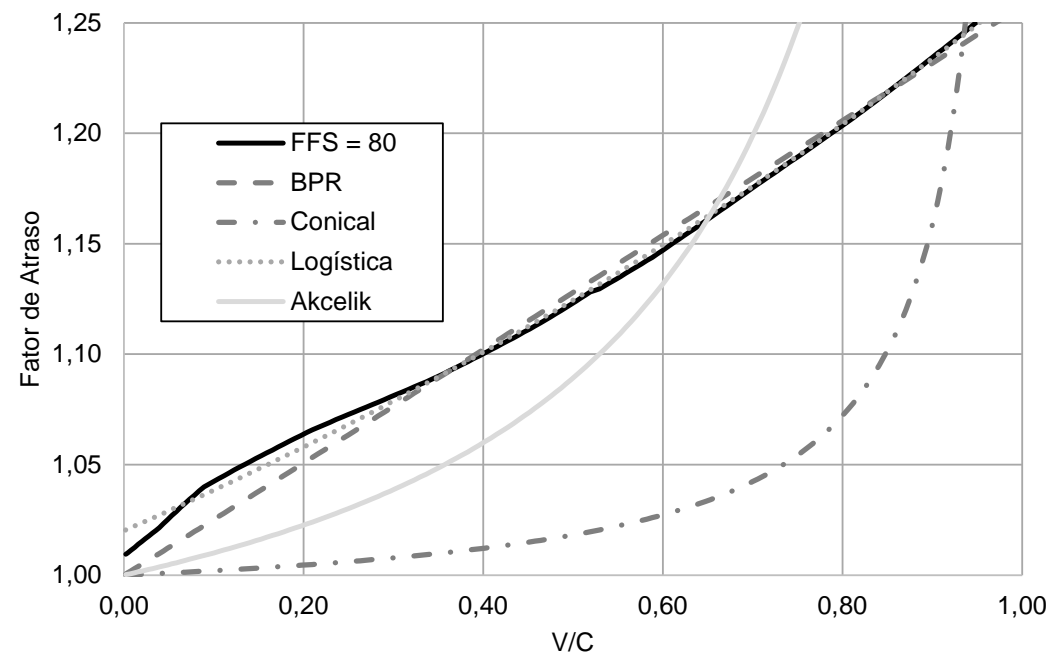

Figura 5. Comparação de funções de atraso para uma rodovia de pista simples de FFS $=80 \mathrm{~km} / \mathrm{h}$ 
Tabela 2. Parâmetros de calibração e erro por VDF e FFS - rodovias de pista simples em terreno genérico

\begin{tabular}{|c|c|c|c|c|c|c|c|c|}
\hline \multirow{2}{*}{$\begin{array}{l}\text { Parâmetro } \\
F F S\end{array}$} & \multicolumn{4}{|c|}{ Rodovias de pista simples - terreno plano } & \multicolumn{4}{|c|}{ Rodovias de pista simples - terreno ondulado } \\
\hline & 110 & 100 & 90 & 80 & 110 & 100 & 90 & 80 \\
\hline \multicolumn{9}{|c|}{ Função do BPR } \\
\hline$\alpha$ & 0,16 & 0,18 & 0,21 & 0,26 & 0,16 & 0,18 & 0,21 & 0,26 \\
\hline$B$ & 1,01 & 1,01 & 1,01 & 1,01 & 1,01 & 1,01 & 1,01 & 1,01 \\
\hline Erro & 0,006 & 0,006 & 0,005 & 0,005 & 0,011 & 0,012 & 0,011 & 0,012 \\
\hline \multicolumn{9}{|c|}{ Função Conical } \\
\hline$\alpha$ & 28,0 & 37,9 & 51,2 & 28,3 & 28,5 & 38,1 & 52,6 & 28,4 \\
\hline Erro & 2,543 & 2,231 & 2,373 & 2,764 & 2,524 & 2,229 & 2,375 & 2,769 \\
\hline \multicolumn{9}{|c|}{ Função de Akçelik } \\
\hline$J$ & 0,9832 & 1,2046 & 1,5059 & 1,9296 & 0,9832 & 1,2046 & 1,5059 & 1,9296 \\
\hline Erro & 5,698 & 5,644 & 5,470 & 5,099 & 5,705 & 5,652 & 5,480 & 5,112 \\
\hline \multicolumn{9}{|c|}{ Função Logística } \\
\hline$C_{1}$ & 0,78 & 0,84 & 0,76 & 0,78 & 0,66 & 0,89 & 0,77 & 0,77 \\
\hline$C_{2}$ & 0,72 & 0,7 & 0,68 & 0,67 & 0,83 & 0,56 & 0,79 & 0,8 \\
\hline$C_{3}$ & 0,75 & 1,12 & 0,57 & 0,66 & 0,31 & 1,2 & 0,82 & 0,83 \\
\hline$C_{4}$ & 0,55 & 0,78 & 0,75 & 0,93 & 0,37 & 0,99 & 0,67 & 0,78 \\
\hline Erro & 0,000 & 0,000 & 0,001 & 0,001 & 0,001 & 0,001 & 0,002 & 0,002 \\
\hline
\end{tabular}

enquanto que a Tabela 3 mostra a mesma análise para aclives-tipo com inclinação variada e $1 \mathrm{~km}$ de extensão. Novamente, as funções do BPR e a logística foram aquelas que produziram o melhor ajuste, em especial a última.

A Figura 5 mostra as VDFs ajustadas para uma rodovia de pista simples típica de FFS $=80 \mathrm{~km} / \mathrm{h}$, em terreno ondulado. Tanto no caso mostrado quanto para uma rodovia similar em terreno plano, o melhor ajuste obtido por meio da função do BPR se dá usando-se $\beta=1,01$ (valor mínimo), o que torna a Equação 1 linear. Vale notar que o uso dos defaults normalmente encontrados na literatura $(\alpha=0,15 \mathrm{e}$ $\beta=4$ ) produz uma relação parabólica tipicamente relacionada a uma rodovia duplicada.

Ajustes melhores são obtidos com o emprego da função logística, visto que esta é capaz de estimar um aumento mais acentuado no atraso para baixos volumes de tráfego, reflexo do uso do $f_{g}$ na equação. Além disso, a função logística também produz maiores atrasos na capacidade e em condições de congestionamento, o que se mostra desejável em uma VDF.

A função Conical, por sua vez, apresenta as mesmas deficiências descritas para o caso das rodovias de pista du- pla. Neste caso porém, tais desvantagens se acentuam, devido ao maior atraso em condições de baixo fluxo de tráfego característico das rodovias de pista simples.

Por fim, a função de Akçelik, é análoga ao próprio modelo do HCM para rodovias duplicadas. Para adaptá-la às rodovias de pista simples, o parâmetro $B P$ da Equação 5 deve ser definido como 0 . Ainda assim, o ajuste obtido é notadamente inferior àquele produzido pelas funções do BPR e Logística.

\subsection{Considerações quanto à aplicabilidade e limitações dos parâmetros propostos}

Os parâmetros propostos neste trabalho foram obtidos por meio da definição de um problema de otimização, no qual os coeficientes de cada modelo testado devem produzir relações as mais próximas o possível com um modelo de referência, neste caso, relações fluxo-velocidade calibradas para rodovias paulistas.

Dessa forma, os resultados atingidos podem ser considerados válidos para segmentos rodoviários com características similares aos usados por Andrade e Setti (2014), que consistiam em autoestradas e rodovias de pista dupla típicas do estado de São Paulo. Para trechos homogêneos distintos,

Tabela 3. Parâmetros de calibração e erro por VDF e FFS - aclives de rodovias de pista simples

\begin{tabular}{|c|c|c|c|c|c|c|c|c|c|c|c|c|}
\hline \multirow{2}{*}{$\begin{array}{l}\text { Parâmetro } \\
\text { FFS }\end{array}$} & \multicolumn{4}{|c|}{ Aclive-3\% } & \multicolumn{4}{|c|}{ Aclive-5\% } & \multicolumn{4}{|c|}{ Aclive - 7\% } \\
\hline & 110 & 100 & 90 & 80 & 110 & 100 & 90 & 80 & 110 & 100 & 90 & 80 \\
\hline \multicolumn{13}{|c|}{ Função do BPR } \\
\hline$\alpha$ & 0,146 & 0,168 & 0,198 & 0,237 & 0,157 & 0,182 & 0,215 & 0,258 & 0,158 & 0,183 & 0,217 & 0,260 \\
\hline$B$ & 1,010 & 1,010 & 1,010 & 1,010 & 1,010 & 1,010 & 1,010 & 1,010 & 1,010 & 1,010 & 1,010 & 1,010 \\
\hline Erro & 0,009 & 0,010 & 0,008 & 0,009 & 0,014 & 0,014 & 0,014 & 0,016 & 0,024 & 0,026 & 0,028 & 0,033 \\
\hline \multicolumn{13}{|c|}{ Função Conical } \\
\hline$\alpha$ & 51,0 & 51,0 & 51,0 & 38,3 & 51,0 & 51,0 & 51,4 & 28,5 & 51,0 & 51,0 & 51,0 & 28,8 \\
\hline Erro & 1,744 & 1,815 & 2,016 & 2,371 & 1,978 & 2,094 & 2,376 & 2,771 & 1,983 & 2,099 & 2,383 & 2,784 \\
\hline \multicolumn{13}{|c|}{ Função de Akçelik } \\
\hline$J$ & 0,9048 & 1,1098 & 1,3887 & 1,7812 & 0,9832 & 1,2046 & 1,5059 & 1,9296 & 0,9832 & 1,2046 & 1,5059 & 1,9296 \\
\hline Erro & 5,437 & 5,452 & 5,364 & 5,111 & 5,709 & 5,657 & 5,486 & 5,120 & $80 \%$ & $80 \%$ & $80 \%$ & $80 \%$ \\
\hline \multicolumn{13}{|c|}{ Função Logística } \\
\hline$C_{1}$ & 0,7467 & 0,8908 & 0,7364 & 0,7372 & 0,6312 & 0,8820 & 0,7189 & 0,6950 & 0,6245 & 0,8432 & 0,6477 & 0,6130 \\
\hline$C_{2}$ & 0,7914 & 0,7405 & 0,8105 & 0,8490 & 0,9294 & 0,6807 & 0,9794 & 1,0474 & 0,9404 & 0,7757 & 1,1105 & 1,2041 \\
\hline$C_{3}$ & 0,6833 & 1,5751 & 0,6620 & 0,7303 & 0,3699 & 1,3792 & 0,8410 & 0,8233 & 0,3505 & 1,2168 & 0,7026 & 0,6870 \\
\hline$C_{4}$ & 0,4320 & 0,8337 & 0,5671 & 0,6454 & 0,3172 & 0,8678 & 0,5101 & 0,5409 & 0,2977 & 0,6815 & 0,3815 & 0,3923 \\
\hline Erro & 0,001 & 0,001 & 0,001 & 0,002 & 0,002 & 0,001 & 0,002 & 0,003 & 0,003 & 0,003 & 0,005 & 0,007 \\
\hline
\end{tabular}


fatores diferentes são esperados. Neste caso, uma calibração local é desejável.

Para tanto, é necessário dispor de observações da taxa de fluxo e velocidade média da corrente de tráfego para intervalos de uma hora ou menos. A partir da velocidade e conhecendo-se a velocidade de fluxo livre da via, deve-se montar gráficos contendo o fluxo versus o atraso. Por fim, sobre esses dados, aplica-se uma técnica de otimização, como citado anteriormente, com o objetivo de minimizar a diferença entre o quadrado dos erros da função escolhida e do modelo de referência.

\section{CONCLUSÕES E RECOMENDAÇÕES}

Neste trabalho, foi analisada uma ampla gama de funções de atraso (VDFs), sendo descritas aquelas mais citadas na literatura e mais comumente usadas nos pacotes de modelagem de demanda por transporte: (1) função do BPR; (2) função Conical; (3) função de Akçelik; e (4) função logística.

A partir da análise da literatura, pôde-se inferir que, em comum, a maior parte das VDFs ou dos valores default sugeridos para seus parâmetros de calibração se relacionam de alguma forma com os modelos fluxo-velocidade apresentados pelo Highway Capacity Manual - HCM para autoestradas (freeways) e rodovias de pista dupla (multilane highways), desde sua segunda edição (HRB, 1965). Assim, neste trabalho foi testada a compatibilidade entre as quatro VDFs descritas e os modelos atuais do HCM, calibrados para as condições das rodovias paulistas por Andrade e Setti (2014) e Bessa Jr. e Setti (2011).

Como principais conclusões, têm-se que os melhores ajustes foram obtidos com o uso da função do BPR e da função logística. Por outro lado, as funções Conical e de Akçelik devem ser empregadas com cautela no ambiente rodoviário brasileiro, em especial em rodovias de pista simples, pois tendem a subestimar o atraso em condições de fluxos de tráfego baixos a médios, comuns em rodovias rurais.

Especificamente com relação às rodovias de pista simples, se comparada à tradicional função do BPR, a função logística se mostrou especialmente interessante, devido a sua capacidade de refletir os atrasos mais acentuados para baixos fluxos de tráfego e, simultaneamente, produzir maiores atrasos em condições de congestionamento, característica desejável em modelagem de demanda.

Para trabalhos futuros, é recomendado o ajuste das VDFs para mais configurações de rampas específicas de rodovias de pista simples, com diferentes valores para $f_{g}$. Além disso, é desejável a análise dos fatores de atraso produzidos em condição de demanda acima da capacidade $(V / C>1)$, caso a caso, de forma a verificar se os valores propostos neste trabalho para o regime não congestionado (curvas calibradas do HCM) não gerem resultados inconsistentes para o regime congestionado.

\section{AGRADECIMENTOS}

Os autores agradecem o apoio financeiro do CNPq, sob a forma de uma bolsa de produtividade em pesquisa.

\section{REFERÊNCIAS}

Akçelik, R. (2003) Speed-Flow Models for Uninterrupted Traffic Facilities. Akçelik \& Associates Pty Ltd, 34 p.

Andrade, G. R.; Rodrigues-Silva, K. C.; Puty Filho, S. A. (2011) Panorama Normativo e Tecnológico da Avaliação Operacional das Concessões Rodoviárias. In: Anais do $7^{\circ}$ Congresso Brasileiro de Rodovias e Concessões - CBR\&C 2011, Foz do Iguaçu, PR (CD-ROM), $14 \mathrm{p}$.

Andrade, G. R.; Setti, J. R. (2014) Speed-Flow Relationship and Capacity for Expressways in Brazil. Transportation Research Circular, v. E-C190, p. 10-25. Disponível em: $<$ http://sites.poli.usp.br/d/ptr2377/14-1707setti.pdf>

Bessa Jr., J. E.; Setti, J. R. (2011) Derivation of ATS and PTSF Functions for Two-lane, Rural Highways in Brazil. In: 6th International Symposium on Highway Capacity and Quality of Service, 2011, Stockholm. Procedia: Social and Behavioural Sciences. Amsterdam: Elsevier, v. 16. p. 282-292. DOI:10.1016/j.sbspro.2011.04.450.

BPR (1964) Traffic Assignment Manual. U.S. Department of Commerce, Urban Planning division, Washington, D.C., EUA.

Caliper (2013) Travel Demand Modeling with TransCAD Version 6.0 User's Guide. Caliper Corporation, EUA, 602 p.

HRB (1965) Highway Capacity Manual. Highway Research Board, Washington, D.C., EUA.

Huntsinger, L. F.; Rouphail, N. M. (2011) Bottleneck and Queuing Analysis. Transportation Research Record, v. 2255, p. 117-124. DOI:10.3141/2255-13.

Kurth, D. L.; van den Hout, A.; Ives, B. (1996) Implementation of "Highway Capacity Manual”-Based Volume-Delay Functions in Regional Traffic Assignment Process. Transportation Research Record, v. 1556, p. 27-36.

DOI: $10.3141 / 1556-05$

Machado, O. F.; Ribeiro, P. C. M. (2003) Calibração da Função de Capacidade do BPR para uma Via Expressa Brasileira. In: Anais do I Rio de Transportes, I Congresso de Ensino e Pesquisa do Estado do Rio de Janeiro, Rio de Janeiro, v. 1, p. 1-12.

Setti, J. R. (2009) Highway Capacity Manual ou Manual de Capacidade Viária? Anais do $6^{\circ}$ Congresso Brasileiro de Rodovias e Concessões - CBR\&C 2009, Florianópolis, SC.

Setti, J. R.; Bessa Jr., J. E.; Egami, C. Y.; Mon-Ma, M. L. (2011) Adaptação do HCM2000 para análise da capacidade e do nível de serviço em rodovias de pista simples no Brasil. Transportes, v. 19, p. 66-78. DOI: 10.14295/transportes.v19i2.510.

Singh, R.; Dowling, R. (2002) Improved Speed-Flow Relationships: Application to Transportation Planning Models. In: Proc. 7th TRB Conference on the Application of Transportation Planning Methods, TRB, National Research Council, Washington, D.C., p. 340-349.

Spiess, H. (1990) Conical Volume-Delay Functions. Transportation Science, v. 24, n. 2, p. 153-158. DOI: 10.1287/trsc.24.2.153. 
TRB (1994) Highway Capacity Manual. Special Report 209 (1994 update). Transportation Research Board, Washington, D.C.

TRB (2000) Highway Capacity Manual 2000. Transportation Research Board, Washington, D.C.

TRB (2010) Highway Capacity Manual 2010. Transportation Research Board, Washington, D.C. 